\title{
Using the reassignment procedure to test object representation in pigeons and people
}

\author{
Jessie J. Peissig • Yasuo Nagasaka • Michael E. Young • \\ Edward A. Wasserman • Irving Biederman
}

Published online: 12 March 2015

(C) Psychonomic Society, Inc. 2015

\begin{abstract}
In four experiments, we evaluated Lea's (1984) reassignment procedure for studying object representation in pigeons (Experiments 1-3) and humans (Experiment 4). In the initial phase of Experiment 1, pigeons were taught to make discriminative button responses to five views of each of four objects. Using the same set of buttons in the second phase, one view of each object was trained to a different button. In the final phase, the four views that had been withheld in the second stage were shown. In Experiment 2, pigeons were initially trained just like the birds in Experiment 1. Then, one view of each object was reassigned to a different button, now using a new set of four response buttons. In Experiment 3, the reassignment paradigm was again tested using the number of pecks to bind together different views of the same object. Across all three experiments, pigeons showed statistically significant generalization of the new response to the nonreassigned views, but such responding was well below that to the reassigned view. In Experiment 4, human participants were studied using the same stimuli and task as the pigeons in
\end{abstract}

\author{
J. J. Peissig $(\varangle)$ \\ Department of Psychology, California State University Fullerton, \\ P.O. Box 6846, Fullerton, CA 92834, USA \\ e-mail: jpeissig@fullerton.edu \\ Y. Nagasaka \\ Brain Science Institute, RIKEN, Wako, Japan \\ M. E. Young \\ Department of Psychological Sciences, Kansas State University, \\ Manhattan, KS, USA \\ E. A. Wasserman \\ Department of Psychology, The University of Iowa, Iowa City, IA, \\ USA \\ I. Biederman \\ Department of Psychology, University of Southern California, \\ Los Angeles, CA, USA

Experiment 1. People did strongly generalize the new response to the non-reassigned views. These results indicate that humans, but not pigeons, can employ a unified object representation that they can flexibly map to different responses under the reassignment procedure.

Keywords Concept learning $\cdot$ Human learning $\cdot$ Object recognition $\cdot$ Perception $\cdot$ Pigeon $\cdot$ Psychophysics

Researchers have long sought to understand conceptual behavior in both humans and non-humans. In many instances, the ideas of "categories," "equivalence classes," and "concepts" are all interrelated within the literature (Zentall, Wasserman, \& Urcuioli, 2014). We can define a category or equivalence class as "a set of things that are treated equivalently to one another" (Murphy, 2010, p. 11). Thus, "tree" is a category that would include such diverse instances as pine trees, oak trees, and apple trees. The concept of a "tree" is often also deemed to be the cognitive representation that we refer to when we view a novel instance of a tree (for example, a gingko) and are able to classify it as a member of the category (Murphy, 2010). However, it is important to be clear that behavioral evidence suggesting that a group of individual instances forms a category for an organism does not tell us much about what the organism knows about that "concept." It is this particular issue that has been of interest to researchers studying concepts in non-humans.

In one of the first studies to explore concepts in pigeons, Herrnstein and Loveland (1964) taught pigeons to discriminate visual scenes that contained humans from those that did not. Their study, as well as others that followed (e.g., Bhatt, Wasserman, Reynolds, \& Knauss, 1988; Mallot \& Siddal, 1972; Siegel \& Honig, 1970), attempted to determine the 
nature of the representation or "concept" that the pigeon acquires in the course of such discrimination learning.

Previous forced-choice tasks have shown that pigeons can readily learn to discriminate single views of four different objects and later generalize that discriminative responding to novel views of those objects (Peissig, Young, Wasserman, and Biederman 2000a), although generalization performance is often significantly below that of the trained views. Peissig, Wasserman, Young, and Biederman (2002) further showed that training with multiple views increases generalization performance. Such increased generalization applied not only to the rotational axis of the trained views (e.g., the horizontal axis), but also to an untrained and orthogonal rotational axis (e.g., the vertical axis). Finally, go/no-go tasks (Peissig, Young, Wasserman, and Biederman 2000b) have further shown that pigeons treat different views of the same object as more visually similar to one another than to different views of different objects. All of these data collectively suggest that pigeons perceive a correspondence among different views of the same object. What we do not know, however, is how these views are structurally related in memory, or how far we can push this correspondence.

The generality of object representations is a particularly important issue, pertaining directly to our understanding of how both humans and non-humans employ object concepts (Biederman, 2001; Peissig \& Tarr, 2007). The data thus far collected suggest that humans and non-human animals may use similar object representations, although there may be some disparities (for a review see Soto \& Wasserman, 2014). For example, Spetch and Friedman (2003) found that humans showed an advantage for interpolated views (between two learned views) versus extrapolated views (not between two learned views). In their study, however, pigeons did not show this same advantage for interpolated versus extrapolated views. In contrast, Peissig et al. (2002) found that pigeons showed better generalization for views from an orthogonal axis, similar to results found with humans (Bülthoff \& Edelman, 1992), if they were trained with multiple views. It is possible that some of the differences found among various studies of object recognition in pigeons may relate to how flexibly the pigeons are able to use their object representation in different conditions.

Martin-Malivel et al. (2006) found that, in a face discrimination task, both humans and baboons could discriminate noisy images of human and baboon faces equally well. However, their evidence further suggests that humans and baboons were using different information to solve the task. Martin-Malivel et al. used classification images to analyze what information each species was utilizing to classify human and baboon faces within noise. Their results using this technique suggested that baboons tended to use coarse image information from the eye region, and they responded in accord with a theoretical observer using only pixel similarities. In contrast, Martin-Malivel et al. found that humans used both coarse and fine visual information from the entire face. Martin-Malivel suggested that baboons may resort to using simple image properties to most efficiently solve the laboratory task, even if they are capable of using more complex information in real-life tasks. Importantly, Soto and Wasserman (2012) were able to take the disparate findings for object categorization in both primates and pigeons and account for all of the results within a single model. Thus, species as distinct from one another as humans, baboons, and pigeons may be using similar underlying object representations, although the extent to which these representations might be flexibly used across diverse tasks might differ.

Astley and Wasserman (1992) used a go/no-go paradigm to see if untrained stimulus similarities, such as those between different views of the same object or between different members of the same category, might be spontaneously exhibited. The results of Astley and Wasserman as well as those of Peissig et al. (2000b) support theories of categorical coherence, which propose that perceptual similarity plays a key role in the formation of categories.

Certainly, perceptual similarity is foundational to stimulus generalization and it likely relates to the organism's underlying neural architecture, particularly if the common features are critical for recognition. For example, Sigala and Logothetis (2002) found that neurons in monkey visual cortex (specifically, the anterior inferior temporal cortex) were highly likely to encode the perceptual features of an object that were relevant in the recognition task. What is unclear, however, is whether the pigeons (or monkeys) in these studies learned something more generic about these object classes or shapes. These studies thus do not unequivocally show that nonhumans form general object concepts similar to the types of concepts that humans are believed to form.

In the current set of experiments, we explored Lea's (1984) innovative reassignment procedure for studying object representation in pigeons. Lea proposed the reassignment procedure as a critical test of conceptual behavior. He argued that many experimental demonstrations of conceptual behavior in pigeons may be attributable to simpler explanations than concept formation. Pigeons may merely have learned that combinations of specific features can be used to discriminate among the training stimuli. This type of feature discrimination could allow the pigeons to generalize their behavior to novel stimuli that share some of the features of the training stimuli. For example, pigeons might look for leaves, bark, or branches to discriminate pictures of trees from pictures of other objects. Even novel instances of trees are likely to contain at least one of these features; thus, the pigeons should generalize their behavior to novel trees.

Lea questioned whether this type of feature discrimination learning should be defined as concept learning. He suggested 
that "something more" may be required to adequately define a concept. In human research, that "something more" has often been proposed as the ability to verbally define or to cognitively manipulate the concept. Indeed, humans (and perhaps other species) appear to be predisposed to create equivalence classes (Zentall et al., 2014).

A recent study by Cimpian and Erickson (2012) suggests that children are predisposed to partition their world into groups of similar "kinds." They found that children were much better at remembering information about particular "kinds" than particular exemplars; for example, children better remembered that "dogs" exhibit a specific behavior than they remembered that a particular dog exhibits that same behavior. These findings indicate that people, even from a very young age, are adept at forming equivalence classes. That is, they may more readily form a class for all "dogs" or all "toys" than just memorizing individual instances.

Despite the evidence that both humans and non-humans behave as if they form equivalence classes, without a direct window into the mind of an individual, it is difficult to know what precisely has been learned. For example, Lea (1984) proposed that even in a more complex experiment such as that of Herrnstein and Loveland (1964), animals may not be using a general concept or equivalence class to solve the discrimination. A task requiring pigeons to peck at images containing people and not to peck at similar images without people could be solved by learning the concept of "person" and looking for a person in an image. Based on studies such as Cimpian and Erickson (2012), that is precisely how we would expect people to perform the task, even from an early age. Alternatively, the pigeons could simply learn specific responses to each image used in the experiment (Lea, 1984, pp. 265-266). This type of stimulus-response learning is not what we would consider general concept learning of that same kind as is believed to be characteristic of human categorization. Consequently, additional behavioral evidence is necessary to determine the nature of the representation used by non-humans to perform these categorization tasks.

To test for that "something more" of concept formation in non-human animals, Lea outlined a three-step experimental design. In Step 1, the animal learns a concept in the usual way. For example, the pigeon learns to peck at pictures of trees and to withhold pecks to pictures of fish. In Step 2, the animal learns to associate a new response to a subset of the exemplars that comprise the learning set of each concept (in this case, trees and fish). In the experimental example given by Lea (1984), the trained response in Step 2 is a reversal of the response learned in Step 1; so, the pigeon is now taught to peck to a subset of the pictures of fish and to withhold pecks to a subset of the pictures of trees. In Step 3, the animal is tested with those exemplars of the concepts that were not shown in Step 2; in keeping with the example, this would mean that the pigeon should now peck at these fish and to withhold pecks to these trees, even though these particular stimuli had only been shown in Step 1 when they occasioned the opposite response tendencies.

According to Lea, this type of experiment should provide a better test of concept formation in non-human animals. However, the failure to find a positive result has two possible explanations. First, a negative result using Lea's procedure could indicate that pigeons do not form a general category or object concept that they can cognitively manipulate. Instead, pigeons may simply form networks of associations among the features that are likely to be associated with the category or object. Humans may also sometimes form concepts in this way, but we presume that humans are also able to form more general concepts, as defined above. Second, a negative result may indicate that Lea's suggested method for testing concepts in animals is unable to sensitively diagnose these more general concepts in animals, due to limitations in the pigeon's ability to flexibly respond to changes in response contingencies. There may be considerable interference between what is learned in Steps 1 and 2 , thereby confusing the animal by the time it is finally tested in Step 3 (Zentall, 2012).

Several studies have used some version of the reassignment procedure to study concept formation in non-humans, with varying degrees of success. For example, Vaughan (1988) found positive transfer in a study looking at the formation of equivalence classes in pigeons. Vaughan's results suggest that, at least under some conditions, pigeons are able to form equivalence classes. However, he used numerous reversals and reinforcement contingencies that were directly associated with these classes (the sets of stimuli were reversed from $\mathrm{S}+$ to $\mathrm{S}^{-}$).

Similarly, Wasserman, DeVolder, and Coppage (1992) explored concept formation in pigeons using categories that were not visually similar to try to create equivalence classes in pigeons. The pigeons exhibited significant transfer to the untrained stimuli, making it evident that the pigeons had formed equivalence classes even with non-similarity-based categories; specifically, pigeons chose the same response for the cars as they did for the people if those categories had been associated with the same response in the first phase of training. Similar to Vaughan (1988), these data suggest that pigeons can form a general concept related to a common reinforcement contingency using a form of the reassignment procedure.

In accord with Vaughan (1988) and Wasserman, DeVolder, and Coppage (1992), Delius, Ameling, Lea, and Staddon (1995) found evidence for the formation of equivalence classes in pigeons using a version of the reassignment procedure as well. Although their transfer effects were not large, they were significant and provide more evidence that the reassignment procedure can be used to test for the formation of a concept based on common reinforcement contingencies.

In contrast, there are other studies that have been unable to successfully demonstrate instance to category generalization 
as proposed by Lea (1984). For example, Von Fersen and Lea (1990) did not obtain uniformly positive results. In their study, the authors trained common responses to two groups of stimuli. The responses were then reversed for a subset of the two groups. Finally, pigeons were tested with group members that either shared features with those members given reversal training or did not share features with those given reversal training. Von Fersen and Lea argued that in order to show evidence of having formed a true "concept," pigeons should generalize the response reversal to members that do share features and that do not share features with the trained group members. They found no evidence that pigeons generalized the reversal to stimuli that did not share features in common with the stimuli given reversal training.

Bhatt and Wasserman (1989, Experiment 2) used a version of Lea's reassignment technique to determine whether pigeons formed equivalence classes for perceptual categories of objects and were unable to demonstrate concept formation using the reassignment paradigm. Bhatt and Wasserman (1989) reported that the pigeons' performance in the reassignment task suggested that they may not have formed an equivalence class. Alternatively, the reassignment paradigm may simply be an inadequate test of equivalence classes in pigeons, because the correct choice during testing was ambiguous (Zentall, 2012). Indeed, without explicit instructions, a human participant in this sort of task may not be sure whether the experimenter expects him or her to choose the old or new response during testing.

Given the mixed results obtained in previous studies, it is unclear whether the negative results indicate the pigeon's inability to adequately adjust to the changing response contingencies used in reassignment training or if these failures reflect a true representation of how these classes are instantiated in the animal's brain. In the present study, our goal was to assess the reassignment procedure as a technique for studying object representations in pigeons and, more importantly, to better understand how objects are represented in the pigeon brain.

Wasserman and colleagues tested for category formation using a wide variety of procedures (Astley, Peissig, \& Wasserman, 2002; Astley \& Wasserman, 1992, 1998, 1999; Bhatt \& Wasserman, 1989; Wasserman \& Bhatt, 1992); the reassignment procedure was the only one to fail (Bhatt \& Wasserman, 1989). In the hope of providing a more sensitive assessment of the reassignment method in the present study, we deployed it in a setting that involves a particularly basic form of categorization: generalizing across large changes (e.g., $\leq 72^{\circ}$ of depth rotation) in views of the same object, where the competing stimuli were different objects, varying in non-accidental properties (NAPs; Biederman, 1987). It is possible that pigeons may have an easier time adjusting to changing contingencies if the task itself is simpler, thereby potentially decreasing the birds' cognitive load.
We chose very simple objects for our study: geons. Geons are defined as basic object shapes corresponding to simple parts which, along with their spatial configuration, make up the general representation of an object. This componential representation is similar to the way letters (or spelling patterns) in a particular order make up words (Biederman, 1987). Non-accidental properties are those characteristics, such as symmetry and whether an axis is curved or straight, that are true indicators of the real-world shape of a part, in that they are invariant with orientation in depth, for example a contour that is curved will remain curved under all viewpoints except when its axis of curvature is aligned with the viewpoint, as when the image of the edge of a penny appears as a two-dimensional rectangle. In contrast, an accidental property, such as the degree of curvature or the angle of attachment of two parts, would be a characteristic that varies with viewpoint and is thus, in general, not an invariant characteristic of the object.

In the current study, generalization by pigeons of the reassigned response to the withheld object views would provide clear evidence that pigeons do form general object representations for each object, with the representations specifying NAPs, similar to those proposed by Biederman (1987). If, however, the pigeons do not show generalization of the reassigned response, then that may indicate that pigeons do not form general object concepts and instead store individual object views that can be either be strongly or loosely associated with one another to form an "object" concept.

\section{Experiment 1}

In Experiment 1, we tested the flexibility of the pigeon's object representation by first training birds with five different views of each geon in order to encourage their attending to the relevant stimulus features of each object. The pigeons were next trained to make a new response to only one of those views (reassignment training). If this training successfully accesses the pigeons' object representations, then when the birds are finally given testing trials with the four withheld views, they should be inclined to make the new response, which would be consistent with the formation of a general viewpoint-invariant representation. Alternatively, the pigeons may make the old response learned during original training or make a variety of responses, both old and new; these alternative outcomes would be consistent the idea that the pigeons had stored individual representations of specific object views which are not strongly associated with one another.

Method

Participants The participants were four feral pigeons maintained at $85 \%$ of their free-feeding weights. Prior to the 
present experiment, the pigeons had participated in unrelated studies.

Apparatus The pigeons were trained in four specially constructed plywood chambers. One side of each chamber consisted of a large opening with an aluminum frame attached to the outside; inside the frame was a clear touch screen (AccuTouch ${ }^{\circledR}$ Model \#002744-FTM-K1; Elographics, Oak Ridge, TN) that was coated with mylar for durability. The pigeons' pecks to the touch screen were processed by a serial controller board (Model \#E271-2210, Elographics, Menlo Park, CA). A brushed aluminum panel was placed directly in front of the screen to allow the birds access to limited portions of the video monitor. There were five openings in the aluminum panel: the center opening was a 7-cm $\times 7-\mathrm{cm}$ square opening in which the visual stimuli appeared, and the remaining four openings were circular, $1.9 \mathrm{~cm}$ in diameter, and were located $2.3 \mathrm{~cm}$ from each corner of the center display opening (see Fig. 1). The four corner openings served as report buttons. In the rear of the chamber, a clear Plexiglas food cup was placed level with a wire mesh floor to prevent the pigeons from perching on the cup. Noyes 45-mg pigeon pellets were delivered through a vinyl tube into the cup via a rotary pellet dispenser (Model \#ENV-203M; MED Associates, Lafayette, IN). During experimental sessions, illumination of the chamber was provided by a houselight mounted on the upper rear wall of the chamber. A digital I/O interface board (National Instruments Model \#NB-DIO-24, Austin, TX, USA) controlled the pellet dispenser and the houselight.

Control of peripheral stimuli (via the I/O interface) and recording of pigeons' responses (via the serial controller board) were accomplished by four Apple Macintosh ${ }^{\circledR} 7100 /$ 66 Power PC computers. The pigeon's monitor and an

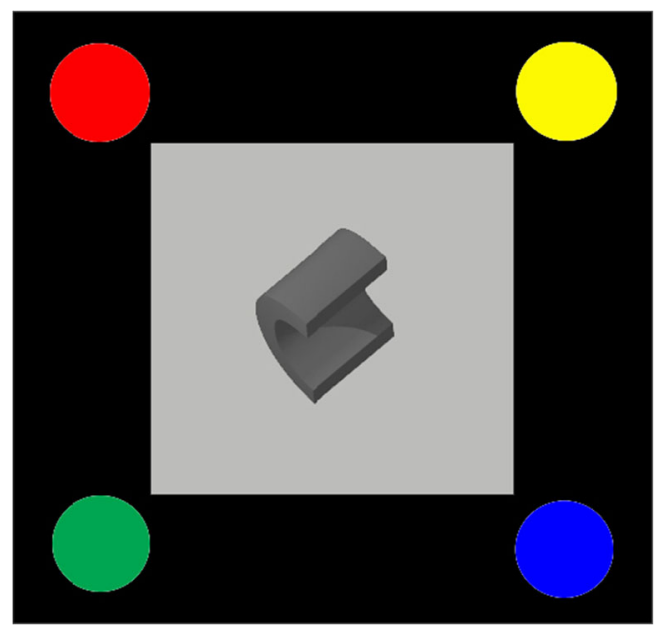

Fig. 1 A bird's eye view of the response panel. The response buttons are placed at the corners of the stimulus display area. The black area shows the placement of the metal panel blocking all other parts of the touchscreen and computer monitor identical monitor located in an adjacent room were connected by a distribution amplifier (Model \#MAC/2 DA2; Extron Electronic, Santa Fe Springs, CA, USA). The programs were developed in HyperCard Version 2.3 (Apple Computer, Cupertino, CA, USA).

Stimuli The stimuli comprised an arch, a barrel, a brick, and a wedge that varied from one another by a variety of nonaccidental properties of their generalized cone characterization (Biederman, 1987; see Fig. 2). The arch had a curved axis and a straight cross-section with parallel sides. All of the other stimuli had straight axes: the barrel had a circular crosssection and non-parallel sides (which meant that the crosssection changed sides as it was swept along the axis); the brick had a square cross-section and parallel sides; the wedge had a straight cross-section and non-parallel sides (Biederman, 1987). The geons were rotated in depth along a horizontal axis in $72^{\circ}$ intervals to yield five different views of each geon (see Fig. 2). The stimuli ranged from 2 to $4 \mathrm{~cm}$ in width and height; they were rendered in Raydream Designer 4 at 300 dots per inch (dpi) resolution, and were shown to the pigeons at $230 \times$ 230 pixels in HyperCard 2.3.

Procedure At the beginning of a trial, the center display area was illuminated with a black cross centered on a white background. A single peck anywhere within that display area turned on a single-geon stimulus in the center. The pigeons were required to peck the center screen a fixed number of times; the number of required pecks was individually adjusted for each bird, depending on its level of performance (for three birds it was 15 pecks and for one bird it was 10 pecks). Completing the final peck illuminated the four corner report buttons (see Fig. 1). After a correct choice, the stimulus was removed from the display area, the report buttons were darkened, and a food pellet reinforcer was delivered. After an incorrect choice, the stimulus was turned off for $1 \mathrm{~s}$, the report buttons were darkened, and the houselight was turned off for from 4 to $6 \mathrm{~s}(M=5 \mathrm{~s})$. The pigeon then proceeded through one or more correction trials (repeating the incorrect trial until a correct choice was made). There was no limit on the number of correction trials that pigeons were given and these trials were not scored for analysis. Intertrial intervals ranged from 6 to $10 \mathrm{~s}(M=8 \mathrm{~s})$.

Experiment 1 comprised three phases: original training, reassignment training, and testing. In original training, the pigeons were taught to peck one of the four different corner report buttons in response to all five views $\left(0^{\circ}, 72^{\circ}, 144^{\circ}\right.$, $-144^{\circ}$, and $-72^{\circ}$, created by rotating the objects in depth in the horizontal axis) of each of the four single-geon stimuli. Button assignments were counterbalanced across the four pigeons using a Latin-square design. We used a randomized block design for original training; each block consisted of one presentation of each of the five views of each of the four 


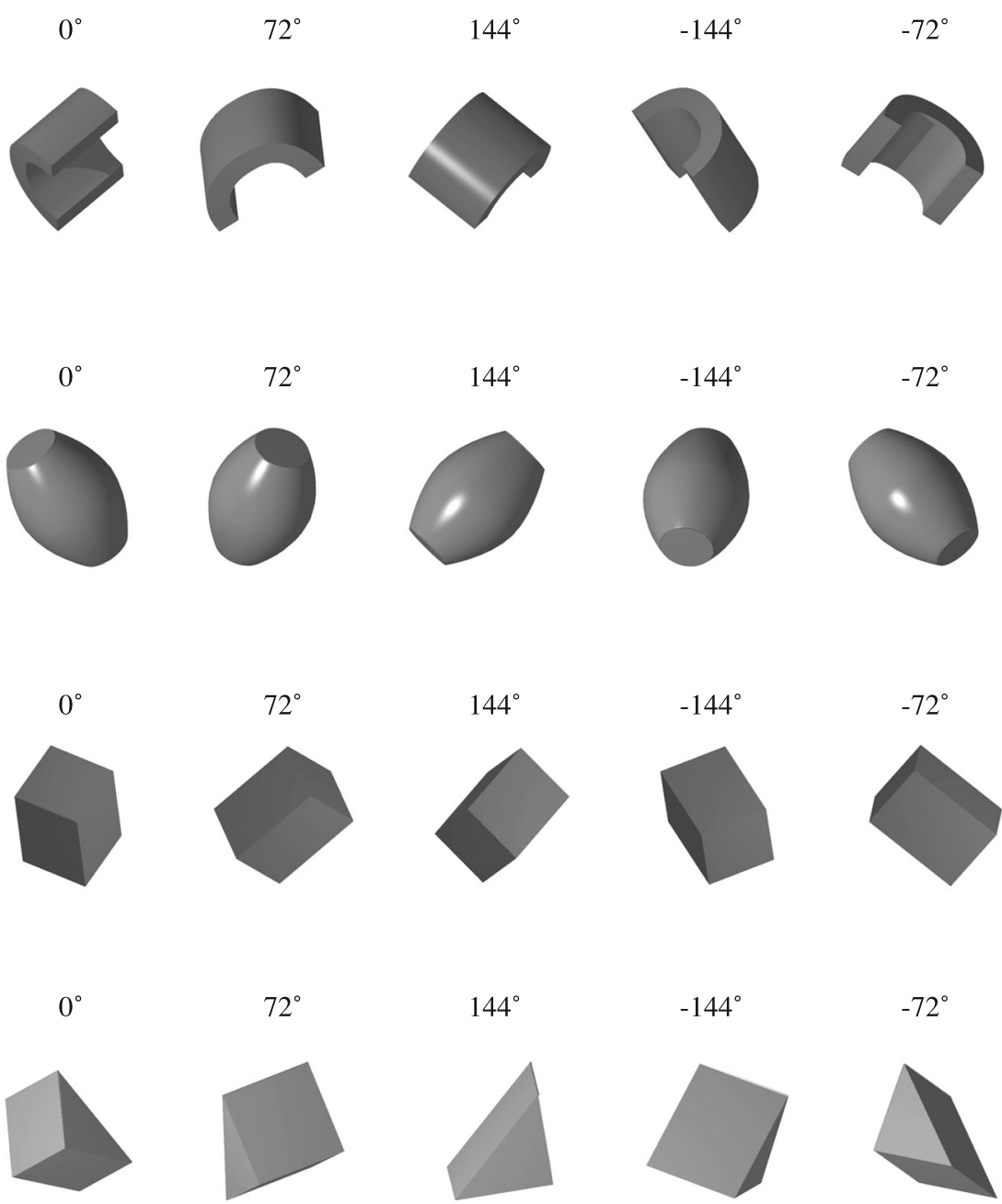

Fig. 2 The $0^{\circ}, 72^{\circ}, 144^{\circ},-144^{\circ}$, and $-72^{\circ}$ views of the arch, barrel, brick, and wedge geons. The $0^{\circ}$ views were used in Reassignment Training

geons, yielding a total of 20 trials. There were ten blocks in each daily session for a total of 200 trials. Each bird had to meet an $85 / 80$ criterion; this criterion meant that a pigeon had to obtain $85 \%$ or above correct as the overall average of all the stimuli and at least $80 \%$ correct for each of the four geons. This criterion assured that pigeons were achieving a high level of performance overall as well as for each object before moving to the next phase. Each bird was required to meet this $85 /$ 80 criterion for a minimum of 1 day before it could proceed to reassignment training.

In reassignment training, one view (the $0^{\circ}$ view) of each of the four geons was reassigned to a new report button. This procedure allowed us to compare these data to previously reported data in which pigeons were trained with only the $0^{\circ}$ view and then immediately tested for generalization to novel views (Peissig et al. 2000a, Experiment 2). The four remaining views of each geon were withheld during reassignment training. Button reassignment was again counterbalanced across the four pigeons. For reassignment training, we used a randomized block design; each block consisted of five presentations of the $0^{\circ}$ view of each of the four geons for a total of 20 trials in a block. There were ten blocks in each daily session for a total of 200 trials. Each bird had to meet the $85 / 80$ criterion for at least 1 day before it could proceed to testing.

In testing, trials with the tested stimuli were nondifferentially reinforced. All of the training trials involved differential food reinforcement; if a pigeon failed to meet the $85 / 80$ criterion for the training trials by the end of a testing session, then it was returned to training for later sessions until it met the $85 / 80$ criterion. The testing stimuli comprised the four views of each of the four geons that were withheld during reassignment training, yielding 16 testing stimuli. In addition, the $0^{\circ}$ views that received reassignment training were also given as testing stimuli during testing sessions, so that there were equal numbers of non-differentially reinforced testing trials for all five views of each geon. This procedure yielded a total of 20 testing stimuli $\left(0^{\circ}, 72^{\circ}, 144^{\circ},-144^{\circ}\right.$, and $-72^{\circ}$ rotations for each of the four geons).

Testing sessions began with eight warm-up trials (two of each training stimulus from reassignment training). The 
remainder of the testing session followed a randomized block design. Each block contained 22 trials involving each of the four reassignment training stimuli appearing five times (20 trials) plus two of the testing stimuli; the two testing stimuli were chosen randomly, without replacement, from the total pool of 20 testing stimuli. There were ten blocks in each daily session for a total of 228 trials (eight differentially reinforced warm-up trials involving the $0^{\circ}$ views, 200 differentially reinforced training views, and 20 non-differentially reinforced testing stimuli). Each pigeon continued until it was tested 20 times with each of the testing stimuli (20 testing sessions).

Results and discussion

The pigeons spent an average of 29 days in original training and 15 days in reassignment training. During testing, they required a mean of 12 days of additional training sessions. Figure 3 shows the mean choice accuracy in testing for Experiment 1 and for a group of pigeons trained with only one view (Control Group; Peissig et al. 2000a, Experiment 2). The data are collapsed across the $-72^{\circ}$ and $72^{\circ}$ views, and across the $-144^{\circ}$ and $144^{\circ}$ views for all four geons; only the non-differentially reinforced trials of the $0^{\circ}$ views were included in both Fig. 3 and the analyses. Chance performance is $25 \%$. The pigeons chose the new, reassigned response significantly more often than would be expected by chance for all of the withheld views (one-tailed binomial, $p<0.05$ ). However, the percentage of times the birds made the reassigned response for the withheld views (35\%) was much lower than for the reassigned view $(82 \%)$.

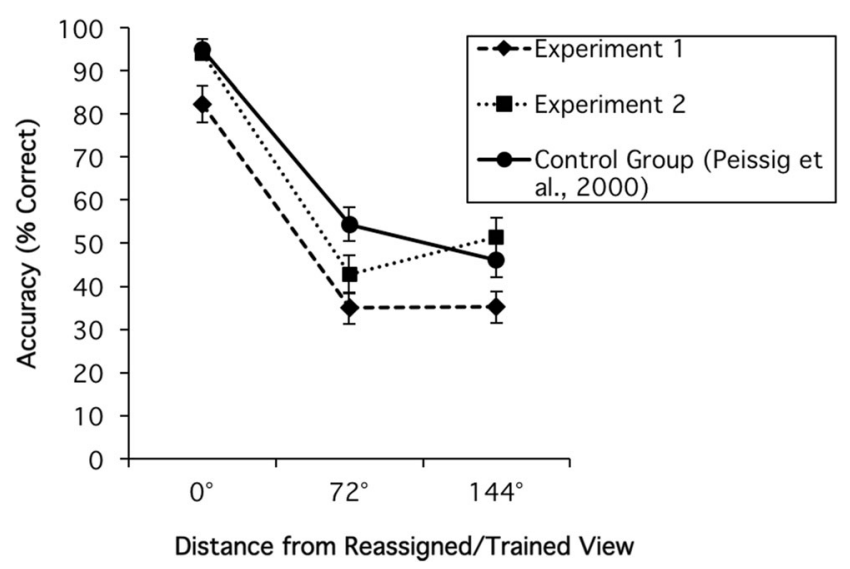

Fig. 3 The choice accuracy scores for Experiments 1 and 2 for the testing phase plotted along with control data from Peissig et al. (2000a, Experiment 2). The data are plotted as a function of distance from the trained/reassigned view $\left(0,72\right.$, and $144^{\circ}$ from the trained view). In Experiment 1 , the button response was reassigned using the same set of buttons from original training. In Experiment 2, reassignment was conducted using a completely new set of buttons (see Fig. 5). The control data were collected from pigeons trained with only a single view of each geon, and then tested with novel views $72^{\circ}$ and $144^{\circ}$ away from the trained view. Error bars represent $95 \%$ confidence intervals
In order to confirm these initial observations, we analyzed choice accuracy using a repeated measures, full factorial $4 \times 5$ ANOVA of Geon (arch, barrel, brick, and wedge) by View $\left(-144^{\circ},-72^{\circ}, 0^{\circ}, 72^{\circ}\right.$, and $\left.144^{\circ}\right)$. We used only the nondifferentially reinforced $0^{\circ}$ views, allowing for an equal number of trials for each view. There was a statistically significant main effect of Geon $\left[F(3,9)=6.54, p=0.01, \eta_{\text {partial }}^{2}=0.08\right]$, indicating that the pigeons exhibited different levels of accuracy to the four different geons (see Table 1). The main effect of View $\left[F(4,12)=19.94, p<0.0001, \eta_{\text {partial }}^{2}=0.20\right]$ was significant, indicating that the pigeons' choice accuracy varied significantly across views. Tukey's HSD test revealed that the comparisons between the $0^{\circ}$ view and the other views $\left(-144^{\circ}\right.$, $-72^{\circ}, 72^{\circ}$, and $\left.144^{\circ}\right)$ were statistically significant $(\alpha=0.05 ; p$ $<0.001)$; the comparisons among the other views were not statistically significant $(\alpha=0.05 ; p>0.05)$. This analysis clearly shows that the difference between the non-reassigned views $\left(-144^{\circ},-72^{\circ}, 72^{\circ}\right.$, and $\left.144^{\circ}\right)$ and the reassigned view $\left(0^{\circ}\right)$ is driving this effect. The interaction of Geon by View was also statistically significant $[F(12,36)=6.52, p<0.0001$, $\left.\eta_{\text {partial }}^{2}=0.12\right]$; this interaction disclosed that the pigeons exhibited different patterns of responding to different views of the four different geons.

The pigeons were tested for a total of 20 sessions, interspersed with Reassignment training if performance fell below criterion. It is possible that this extended testing (which included testing trials with non-differential reinforcement) may have depressed performance, hiding an initial high level of generalization within the first few days of the testing phase. To test this possibility, we analyzed just Days 1 and 2 of the testing phase to determine if the pattern of results was any different. Figure 4 shows the first 2 days of testing plotted along with the full 20 days of testing. It is evident that performance was higher in the first 2 days compared to the full 20 days of testing; however, the pattern of results was the same. To confirm this point, we analyzed choice accuracy for testing phase Days 1 and 2 using a repeated measures, full factorial $4 \times 5$ ANOVA of Geon (arch, barrel, brick, and wedge) by View $\left(-144^{\circ},-72^{\circ}, 0^{\circ}, 72^{\circ}\right.$, and $\left.144^{\circ}\right)$. We found a statistically significant main effect of Geon $[F(3,9)=4.98$, $\left.p=0.05, \eta_{\text {partial }}^{2}=0.14\right]$, indicating that the pigeons exhibited different levels of accuracy to the four different geons. The main effect of View $\left[F(4,12)=8.01, p<0.01, \eta_{\text {partial }}^{2}=0.32\right]$ was significant, indicating that the pigeons' choice accuracy varied significantly across views. The interaction of Geon by View was also statistically significant, $[F(12,36)=2.08, p<$ $\left.0.05, \eta_{\text {partial }}^{2}=0.20\right]$. These were the same outcomes that we found when analyzing all 20 days of data.

The results of Experiment 1 show that the pigeons did exhibit some generalization of discriminative responding to the new, trained response when shown the withheld views (Fig. 3); nevertheless, this generalization was small in magnitude (only $10 \%$ higher than chance). The pigeons did choose 
Table 1 A comparison of the percentage of the total responses for each view consistent with the reassigned response, the percentage of responses consistent with the old, learned response, and the percentage of responses not consistent with either reassignment or original training

\begin{tabular}{|c|c|c|c|c|}
\hline \multirow[b]{2}{*}{ Geon } & \multirow[b]{2}{*}{$\begin{array}{l}\text { Non-reassigned } \\
\text { Views }\end{array}$} & \multicolumn{3}{|c|}{ Percent of total responses } \\
\hline & & $\begin{array}{l}\text { In accord with } \\
\text { reassignment } \\
\text { training }\end{array}$ & $\begin{array}{l}\text { In accord with } \\
\text { original training }\end{array}$ & $\begin{array}{l}\text { Responses not in accord } \\
\text { with either type of training } \\
\text { (random) }\end{array}$ \\
\hline \multirow[t]{4}{*}{ Arch } & -144 & $8.8 \%$ & $55.0 \%$ & $36.3 \%$ \\
\hline & -72 & $11.3 \%$ & $46.3 \%$ & $42.5 \%$ \\
\hline & 72 & $10 \%$ & $21.3 \%$ & $68.8 \%$ \\
\hline & 144 & $26.3 \%$ & $36.3 \%$ & $37.5 \%$ \\
\hline \multirow[t]{4}{*}{ Barrel } & -144 & $51.3 \%$ & $7.5 \%$ & $41.3 \%$ \\
\hline & -72 & $77.5 \%$ & $3.8 \%$ & $18.8 \%$ \\
\hline & 72 & $36.3 \%$ & $11.3 \%$ & $52.5 \%$ \\
\hline & 144 & $31.3 \%$ & $7.5 \%$ & $61.3 \%$ \\
\hline \multirow[t]{4}{*}{ Brick } & -144 & $52.5 \%$ & $16.3 \%$ & $31.3 \%$ \\
\hline & -72 & $30.0 \%$ & $25.0 \%$ & $45.0 \%$ \\
\hline & 72 & $43.8 \%$ & $16.3 \%$ & $40.0 \%$ \\
\hline & 144 & $52.5 \%$ & $15.0 \%$ & $32.5 \%$ \\
\hline \multirow[t]{4}{*}{ Wedge } & -144 & $10.0 \%$ & $1.3 \%$ & $88.8 \%$ \\
\hline & -72 & $15.0 \%$ & $0 \%$ & $85.0 \%$ \\
\hline & 72 & $56.3 \%$ & $3.8 \%$ & $40.0 \%$ \\
\hline & 144 & $48.8 \%$ & $11.3 \%$ & $40.0 \%$ \\
\hline \multicolumn{2}{|c|}{ mean across all geons } & $35.0 \%$ & $17.3 \%$ & $47.6 \%$ \\
\hline
\end{tabular}

the old response for some of the withheld views, but the percentage of choices to the old response button (17\%) was much smaller than was the accuracy achieved in original training (last day of training $=88 \%$ correct).

The pigeons' testing responses can actually be divided into three categories: (1) new, reassigned responses (from reassignment training); (2) old, trained responses (from original

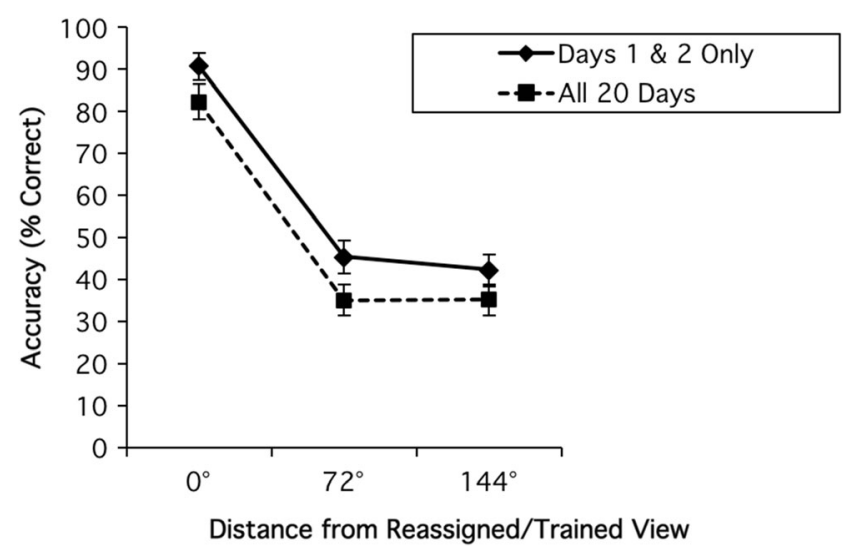

Fig. 4 The choice accuracy scores for days 1 and 2 for the testing phase of Experiment 1 plotted along with the accuracy scores for days 1-20 for the testing phase. The data are plotted as a function of distance from the reassigned view (the $0^{\circ}$ view includes only the non-differentially reinforced trials of that view). Error bars represent $95 \%$ confidence intervals training); and (3) random responses (those to the two report buttons that were never associated with the particular testing object). Categories 1 and 2 explicitly relate to the type of training given, whereas Categories 2 and 3 tell us more about which buttons pigeons prefer to peck when they choose something other than the new, reassigned response.

From Table 1, we can see that, in general, the pigeons were twice as likely to choose the reassigned button compared to the original trained button. In addition, if we look at the percentage of responses to the original trained button compared to the percentage of responses to buttons that were never associated with the testing stimulus, the mean percentage of responses was relatively equally divided, with a slight bias toward choosing the never-associated buttons (17 \% to the one originally trained button, $48 \%$ to the two remaining buttons). Looking more closely at specific geons and views, there is some indication that the pigeons may have defaulted to the original trained response for certain geons (e.g., the arch). However, given the relatively small number of subjects (four) and the predisposition for pigeons to have a response bias for a "default" button whenever they were uncertain how to respond, these data should be interpreted with caution. Indeed, for the wedge, the pigeons were very likely to choose one of the two random buttons, even though they were never associated with that particular geon. 
The accuracy of responses made to the originally trained button was analyzed using a repeated measures, full factorial ANOVA of Geon (arch, barrel, brick, or wedge) by Nonreassigned view $\left(-144^{\circ},-72^{\circ}, 72^{\circ}\right.$, and $\left.144^{\circ}\right)$. The interaction of Geon by Non-reassigned view was significant, $[F(8,26)=$ $3.62, p<0.01, \eta_{\text {partial }}^{2}=0.09$ ], suggesting that the pigeons' likelihood of reverting to the old response for the withheld views varied among the different rotations of each geon. No other effects were significant. This analysis confirms the results presented in Table 1; although there was considerable variability in the percentage of original responses to the different non-reassigned views across the four geons, we did not see an overall trend indicating that pigeons overwhelmingly chose the old response for any particular view or for any specific geon.

As an additional way to evaluate the pigeons' performance in the present experiment, in Fig. 3, we also graphed the results of a previously published experiment in which pigeons were trained with only one view of each object and then tested with novel views (Peissig et al. 2000a; Experiment 2 ). The pigeons trained with the reassignment procedure exhibited very similar generalization to the withheld views to pigeons for which the views were completely novel. Pigeons trained with only one view chose the correct response for that trained view $95 \%$ of the time; they showed moderate generalization to untrained views: $54 \%$ correct at $72^{\circ}$ and $46 \%$ correct at $144^{\circ}$. Thus, the findings of the present reassignment experiment are surely not what one would have expected if the pigeons had generalized the reassigned view's new response to the withheld views.

Recall that we suspected that this experiment would yield one of three possible sets of results for pigeons' responses to the withheld views. Pigeons might make the new, reassigned response on a large percentage of trials. Figure 3 clearly shows that this was not the case. Although the pigeons did choose the new response more than would be expected by chance, they did not choose the new response on a large percentage of trials. Second, pigeons might continue to make the original response to the withheld views. This outcome, however, was not the case; the pigeons chose the old response no more often than they chose buttons that had never been associated with the particular object (approximately $17 \%$ of the time for the old response, and $48 \%$ for both the nonassociated buttons). Third, pigeons might respond with some new responses, some old responses, and some random, incorrect responses. This final possibility appears to be the most suitable summary of the data. The pigeons chose the new response $35 \%$ of the time, which although above chance, is not an especially strong indicator that the pigeons had formed a general object concept for each object.

\section{Experiment 2}

In Experiment 1, our pigeons appear not to have retained much information about the general shape of an object over the course of the entire experiment. One key feature of this experiment was that the four response buttons used in the training phase were the same as those used during the reassignment phase. Thus, the pigeons may have been conflicted as to which response to perform when they were given the withheld views: should they make the new response or the original response? In addition, because the same buttons were used in original training and in reassignment training, we deemed it inappropriate to continue training with the withheld views during the reassignment phase. Consequently, another possible explanation of the pigeon's poor generalization of the new response is that the birds had forgotten the original associative training. Thus, the pigeons may not have formed a general object representation that encompassed all five views. Alternatively, the pigeons did form such a general representation, but it was masked or destroyed by reassignment training. In support of the proposal that the general representation was never formed or was destroyed by training, Fig. 3 shows that the pigeons' performance was similar to the performance of other pigeons that were trained with only one view.

To address these issues, in Experiment 2, pigeons were originally trained with four buttons in the same configuration as in Experiment 1 (Fig. 1). For the reassignment phase, however, a new set of four buttons was offered to the pigeons, which differed in both position and surface from those in the original training (Fig. 5). On any given trial, only one set of buttons was made available. So, during the final phase with the four withheld views, the pigeons could not perform the

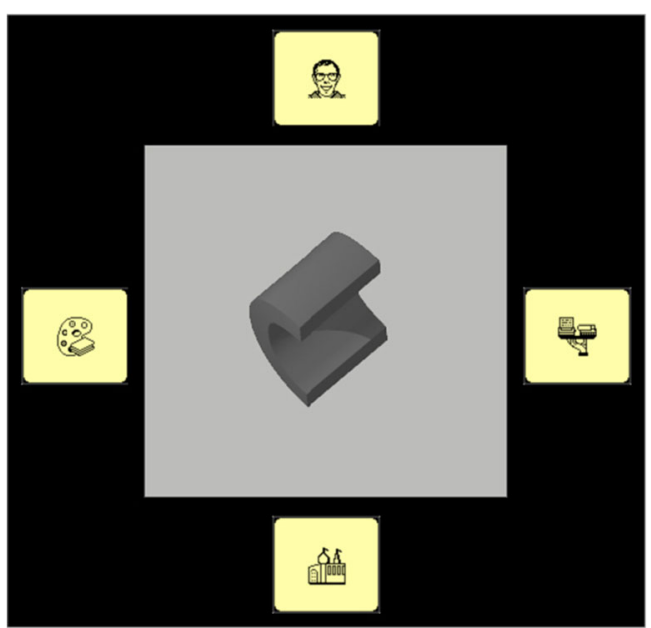

Fig. 5 Schematic representation of the response button placement used for reassignment training in Experiment 2. The black area shows the placement of the metal panel blocking all other parts of the touchscreen and computer monitor. For original training, the buttons were in the same positions as in Experiment 1 (see Fig. 1) 
original responses, presumably making the task demands less ambiguous.

\section{Method}

Participants The participants were four feral pigeons that were different from those studied in Experiment 1. The birds were kept at $85 \%$ of their free-feed weights by controlled daily feeding. The pigeons were placed on a $15-\mathrm{h} / 9-\mathrm{h}$, light/ dark schedule and were given free access to water and grit throughout the experiment. The pigeons had earlier participated in unrelated studies.

Apparatus The pigeons were trained in four operant conditioning boxes detailed by Gibson, Wasserman, Frei, and Miller (2004). The stimuli were presented on a 15-in LCD monitor located behind an AccuTouch ${ }^{\circledR}$ resistive touchscreen (Elo TouchSystems, Fremont, CA, USA). Each chamber was controlled by an Apple ${ }^{\circledR}$ eMac $^{\circledR}$ computer. The experimental procedure was programmed in HyperCard Version 2.4. All other aspects of the apparatus were identical to Experiment 1.

Stimuli The stimuli were the same as those used in Experiment 1, except that they were rendered in Carrara 3 (Eovia, Mountain View, CA, USA) at a resolution $144 \mathrm{dpi}$ and displayed at $240 \times 240$ pixels in Hypercard. Eight black Macintosh icons on light yellow backgrounds served as the report buttons; they were 2.7 centimeters in width and 2.3 centimeters in height. Here, four of the report buttons were placed at the corners of the stimulus window on original training trials; the remaining four report buttons were placed at the top, left, right, and bottom of the stimulus window on reassignment training trials.

Procedure The procedure was nearly identical to Experiment 1 with the exception of the mean length of time for turning off the houselight after an incorrect choice. In Experiment 2, the stimulus, the report buttons, and the houselight were turned off for 5-8 s $(M=6.5 \mathrm{~s})$. The number of required pecks on individual trials was individually adjusted for each bird; in this experiment, it was 7,10 , and 15 pecks for the three pigeons that completed the experiment.

Experiment 2 comprised three phases: original training, reassignment training, and testing. During original training, the pigeons were taught to peck one of the four different corner report buttons in response to all five of the views $\left(0^{\circ}, 72^{\circ}\right.$, $144^{\circ},-144^{\circ}$, and $-72^{\circ}$ ) of each of the four single-geon stimuli. Button assignments were counterbalanced across the four pigeons using a Latin-square design. We used a randomized block design for original training; each block consisted of one presentation of each of the five views of all four geons, yielding a total of 20 trials in a single block. There were ten blocks in each daily session for a total of 200 trials. Each bird had to meet an 85/80 criterion (at least $85 \%$ correct overall and at least $80 \%$ correct for each of the four geons) for at least 1 day before it could proceed to reassignment training.

During the reassignment training phase, we used a randomized block design and each block consisted of two sub-blocks (an original training sub-block and a reassignment training sub-block). The original training sub-block, which was arranged to maintain original training performance, consisted of one presentation of each of the five views of all four geons, yielding a total of 20 trials. The reassignment training subblock consisted of 20 sets of trials. Each set comprised one presentation of the $0^{\circ}$ view of each of the four geons, yielding a total of 80 trials in a single reassignment training sub-block. In the reassignment training phase, the two sub-blocks (an original training sub-block and a reassignment training subblock) were alternated twice in each daily session for a total of 200 trials. Each bird had to meet an 80/85/80 criterion (at least $80 \%$ correct overall for original training trials, $85 \%$ correct overall for reassignment trials, and $80 \%$ correct for each of the four geons on reassignment trials) for at least 1 day before it could proceed to testing. If a pigeon failed to attain at least $80 \%$ correct overall for original training trials by the end of a session, then it was returned to training until it met the $85 / 80$ criterion.

During the testing phase, testing trials were nondifferentially reinforced. All of the training trials and the reassignment trials involved differential food reinforcement. The 16 testing trials comprised four views $\left(72^{\circ}, 144^{\circ},-144^{\circ}\right.$, and $-72^{\circ}$ ) of each of the four geons.

Testing sessions began with 28 warm-up trials: 20 of original training (five views of each of the four geons) plus eight of reassignment training (two blocks of the $0^{\circ}$ view of each of the four geons). The remainder of the testing session followed a randomized block design. Each block contained five trials, with each of the four reassignment training trials appearing once (four trials) plus one of the testing trials. The testing trials were chosen randomly, without replacement, from the total pool of 16 testing stimuli. There were 32 blocks in each daily session for a total of 188 trials (28 differentially reinforced warm-up trials, 128 differentially reinforced reassignment training trials, and 32 non-differentially reinforced testing trials). The pigeons received each testing stimulus twice per session for a total of 20 presentations over the course of ten testing sessions. There was at least one reassignment training session between the testing days. The same criterion from the reassignment phase (80/85/80 criterion) was applied to the testing phase.

\section{Results and discussion}

One of the pigeons was exceedingly slow to learn and did not finish ten testing sessions; therefore, we excluded the data of this bird from our data analysis. The other three pigeons spent 
a mean of 50 days in the original training phase and a mean of 23 days in the reassignment training phase. In the reassignment phase, the pigeons required a mean of 18 days of additional original training. In the testing phase, the pigeons required a mean of 27 days of additional original training and 41 days of additional reassignment training. Only data from the testing phase are presented here.

Figure 3 shows mean choice accuracy in testing (labeled Experiment 2 in the figure). The data are collapsed across the $-72^{\circ}$ and $72^{\circ}$ rotations and the $-144^{\circ}$ and $144^{\circ}$ rotations across all four geons. It is evident from the figure that the pigeons did not exhibit equivalent performance to the reassigned and withheld views. For the reassigned view the pigeons chose the new response $94 \%$ of the time, whereas for the withheld views the pigeons chose the new response much less $\left(72^{\circ}=43 \% ; 144^{\circ}=51 \%\right)$. Although these pigeons were somewhat more accurate than the pigeons in Experiment 1, the pattern of performance was similar in Experiments 1 and 2.

In order to confirm these initial observations, we analyzed choice accuracy using a repeated measures, full factorial ANOVA of Geon (arch, barrel, brick, and wedge) by View $\left(-144^{\circ},-72^{\circ}, 0^{\circ}, 72^{\circ}\right.$, and $\left.144^{\circ}\right)$. The main effect of Geon was not significant $[F(3,6)=2.99, p>0.05]$, indicating that the pigeons' choice accuracy for the different geons did not differ significantly from one another. There was a statistically significant main effect of View $[F(4,8)=48.60, p<0.0001$, $\eta^{2}$ partial $\left.=0.34\right]$, indicating that the pigeons exhibited different levels of accuracy to the five different views. Tukey's HSD test revealed that the comparisons between the $0^{\circ}$ view and the other views $\left(-144^{\circ},-72^{\circ}, 72^{\circ}\right.$, and $\left.144^{\circ}\right)$ were statistically significant; the comparisons among other views were not statistically significant $(\alpha=0.05)$. The interaction of Geon by View was statistically significant $[F(12,24)=3.46, p<0.005$, $\left.\eta_{\text {partial }}^{2}=0.16\right]$; this interaction indicated that the pigeons showed different patterns of responding to the different views of the four different geons.

Just as in Experiment 1, we analyzed Days 1 and 2 of the testing phase to determine if the pattern of results was any different. Figure 6 depicts the first 2 days of testing plotted along with the full 10 days of testing. There was very little difference between performance on the first 2 days of the experiment compared to the full 10 days of testing. We analyzed choice accuracy for testing phase Days 1 and 2 using a repeated measures, full factorial $4 \times 5$ ANOVA of Geon (arch, barrel, brick, and wedge) by View $\left(-144^{\circ},-72^{\circ}, 0^{\circ}, 72^{\circ}\right.$, and $\left.144^{\circ}\right)$. There was no significant main effect of Geon $[F(3,6)=$ $\left.3.32, p>0.05, \eta_{\text {partial }}^{2}=0.14\right]$, indicating similar accuracy across the four different geons. The main effect of View $\left[F(4,8)=34.63, p<0.0001, \eta^{2}\right.$ partial $\left.=0.30\right]$ was significant, indicating that the pigeons' choice accuracy varied significantly across views. The interaction of Geon by View was also statistically significant, $[F(12,24)=2.23, p=0.045$,

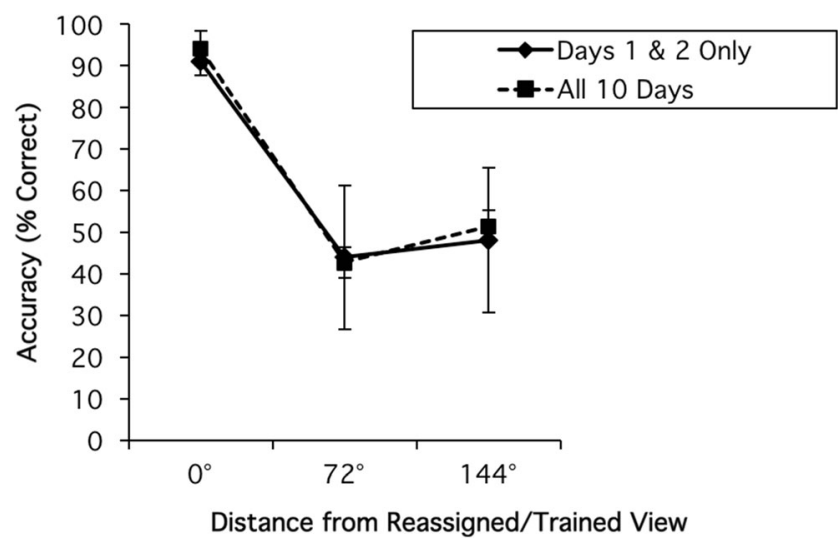

Fig. 6 The choice accuracy scores for days 1 and 2 of the testing phase in Experiment 2 plotted along with the choice accuracy scores for days 1-20 of the testing phase. The data are plotted as a function of distance from the reassigned view. Error bars represent $95 \%$ confidence intervals

$\left.\eta_{\text {partial }}^{2}=0.17\right]$. These were the same outcomes that we found when analyzing all 10 days of data.

These results thus reveal that, even when the old response buttons were made unavailable, the pigeons still exhibited only modest generalization of the newly trained response to the withheld views. The continuation of original training during the reassignment phase of the experiment may have led to slightly better performance overall compared to Experiment 1 . Nevertheless, the pattern of results was the same.

So, it is clear that, using different button report responses, the pigeons did not exhibit evidence of a general object representation using the reassignment paradigm. There are numerous other studies that indicate that pigeons are able to perform well in object recognition tasks (e.g., DiPietro, Wasserman, \& Young, 2002; Peissig et al. 2002; Spetch \& Friedman, 2006), suggesting that even though pigeons may not form a general object concept that would allow them to perform well in the reassignment task, they are able to use individual stored views in a way that allows them to perform well when recognizing objects at different views under some conditions.

\section{Experiment 3}

In Experiments 1 and 2, the nature of the pigeon's visual representation was investigated using reassignment training with different button report responses. In those experiments, we found that the pigeons exhibited some generalization of the new response to the withheld views, but that their response accuracies were significantly lower than their accuracies to the reassigned views. We even found these results despite the use of an entirely different set of response buttons during reassignment training in Experiment 2.

Another possibility is that Experiments 1 and 2 might not have yielded conclusive results due to specific features of the task. Choosing among four different button report responses 
may not be an effective mediating behavior for fusing the different views into a general representation. In an experimental design in which pigeons must retain a complex set of associations over time, the mediation may need to be stronger to be effective.

Astley and Wasserman (1999) showed that experimental paradigms that directly involve the delay or probability of food reinforcement are especially effective in producing mediating associations in pigeons. They trained pigeons with four categories of objects (people, flowers, cars, and people). In their Experiment 1, two categories were associated with a long delay to reinforcement, whereas the remaining two categories were associated with a short delay to reinforcement; in their Experiment 3, pairs of categories were assigned to either a low or a high probability of reinforcement. During choice training, only one category within each pair was trained to a distinctive button response. When the withheld categories of objects were tested on choice trials, the pigeons chose the same button response that was associated with the category that shared a common reinforcement condition (e.g., the same delay to reinforcement). The pigeons responded at a level that was virtually indistinguishable from performance on the trained categories despite the fact that the testing categories had never been directly associated with the response buttons. The success of the delay training procedure is likely to be credited to training the categories using a mediator that directly affected reinforcement. Based on these data, we can surmise that the pigeons had formed strong associations among the objects within the categories based on their initial training; even when a subset of the objects within the category were trained with a new response, the remaining withheld category members were strongly associated with the new response.

Astley and Wasserman (1998) found much poorer generalization when pigeons' original training with all four categories involved an arbitrary button response (nearly a $30 \%$ decrease in accuracy even when the birds were overtrained). Thus, in Astley and Wasserman's (1999) experiments, memory of the equivalence classes formed during the original training phase may have been enhanced by the use of stronger associative "glue" than an arbitrary button response; this is likely to be the result of a stronger salience for responses that directly involve the perceived value of reinforcement. Hence, the pigeons' poor generalization to the withheld views in the present Experiments 1 and 2 may be ameliorated by training with something more effective than single, arbitrary button responses. If the type of mediating event used in original training differentially affects the strength of the mediating associations, then pigeons should be more likely to form and maintain a generalized object representation if we were to use a stronger associative "glue."

To test this hypothesis, we used a modified version of the reassignment paradigm in Experiment 3. Rather than using different button pecks as the mediating responses, the pigeons here had to make a common number of pecks to multiple views of an object. Specifically, different numbers of required pecks were associated with the two presented objects: one necessitated a small work requirement, whereas the other necessitated a large work requirement. Such small and large work requirements are extremely salient to pigeons (Bhatt \& Wasserman, 1987; Felton \& Lyon, 1966).

In Experiment 2, the pigeons appeared not to have retained information about the general shape of an object over the course of the entire experiment. By associating the multiple rotations of each object with a common event that the pigeons find particularly salient - the number of required pecks - the birds in Experiment 3 may be more likely to come under the control of general object shape and to retain this information throughout later stages of the experiment.

Method

Participants The participants were 13 feral pigeons that were different from those studied in Experiments 1 and 2; they were maintained at $85 \%$ of their free-feed weights by controlled daily feeding. Of the 13 pigeons, five did not complete the experiment due to failures in meeting criterion in various phases of the study. The pigeons were kept on a $14-\mathrm{h} / 10-\mathrm{h}$, light/dark schedule and given free access to water and grit throughout the experiment.

Apparatus The apparatus was identical to that used in Experiment 1. Only the center button was used in this experiment.

Stimuli The stimuli consisted of the barrel and brick geons used in Experiment 2 (see Rows 2 and 3 of Fig. 2). These stimuli varied from one another by two non-accidental properties of their generalized cone (Biederman, 1987) characterizations: The barrel had a circular cross-section and nonparallel sides; the brick had a square cross section and parallel sides. The stimuli were rotated in depth by $72^{\circ}$ intervals to yield five different views of each geon. The stimuli ranged from $2-4 \mathrm{~cm}$ in width and from $2-4 \mathrm{~cm}$ in height and were shown in Hypercard at $130 \times 130$ pixels.

Procedure At the beginning of a trial, the center display area was illuminated with a black cross centered on a white background. A single peck anywhere within that display area turned on a single-geon stimulus in the center screen. The pigeons had to peck the center screen a fixed number of times (depending on the stimulus). After the final peck, a food pellet reinforcer was delivered. Intertrial intervals ranged from 6 to $10 \mathrm{~s}(M=8 \mathrm{~s})$.

The experiment consisted of three phases: original training, reassignment training, and testing. Four pigeons were randomly assigned to one of two reinforcement regimens which thus formed a counterbalanced order (eight pigeons total). In 
one regimen, the barrel was associated with 20 pecks and the brick was associated with 60 pecks; in the second regimen, the barrel was associated with 60 pecks and the brick was associated with 20 pecks. Two pigeons in each counterbalancing were randomly assigned to one of two conditions: the Reassignment condition or the Control condition (four pigeons in each).

Pigeons in the Reassignment condition received trials with two single-geon objects shown at five depth rotations: $0^{\circ}, 72^{\circ}$, $144^{\circ},-144^{\circ}$, and $-72^{\circ}$ (see Fig. 2). Pigeons in the Control condition received trials with two single-geon objects shown at one depth rotation, $0^{\circ}$.

In original training, we used a randomized block design. In the Reassignment condition, each block consisted of two presentations of each of the five views of the two objects, for a total of 20 trials in a single block. In the Control condition, each block consisted of ten presentations of the $0^{\circ}$ view of each object, for a total of 20 trials in a single block. Original training comprised eight blocks in each daily session for a total of 160 trials per day.

Performance was measured by response rate (pecks per s), which was defined as the overall rate including the latency to the first peck (Felton \& Lyon, 1966). Pigeons exhibit a strong tendency to peck quickly when a stimulus is associated with a small amount of work and to peck slowly when a stimulus is associated with a large amount of work; this result is largely due to an increase in the latency to begin pecking when presented with a stimulus associated with a large amount of work (Felton \& Lyon, 1966). In order to calculate the peck rate in pecks per second for each trial, we divided the number of total pecks required by the total number of seconds it took for the pigeon to complete the trial after stimulus presentation.

All pigeons began the experiment with a Fixed Ratio of 20 pecks (FR20) and 60 pecks (FR60) as the assigned peck requirements, but we adjusted the higher peck requirement ( 60 pecks) for individual birds as needed to encourage discrimination learning and to maintain discriminative performance (see Table 2 for the peck requirements of individual birds during the testing phase). Each bird was required to remain in original training until the peck rate to the geon associated with FR20 was 1.0 pecks per s higher than the peck rate to the geon associated with FR60 (or whatever the greater peck requirement happened to be for that particular bird). The pigeons had to maintain this performance for 3 consecutive days to meet criterion. For the Reassignment condition, we used the mean peck rate across all five views. This criterion ensured that the pigeons were accurately discriminating between the geons. In addition, requiring the same learning criterion for pigeons in the Reassignment and Control conditions assured that both groups had been trained to an equivalent level of performance, despite the differing proportion of trials with the $0^{\circ}$ view within daily sessions. Pigeons progressed to reassignment training once they had met criterion.
Table 2 Peck requirements during the testing phase for pigeons in Experiment 3

Individual pigeon peck requirements

\begin{tabular}{|c|c|c|}
\hline $\begin{array}{l}\text { Birds in the experimental } \\
\text { condition }\end{array}$ & Peck requirement & Days on requirement \\
\hline $31 \mathrm{~B}$ & 20,140 & 20 \\
\hline \multirow[t]{3}{*}{$23 \mathrm{Y}$} & 20,80 & 14 \\
\hline & 20,75 & 3 \\
\hline & 20,60 & 3 \\
\hline \multirow[t]{2}{*}{$63 \mathrm{~W}$} & 20,80 & 18 \\
\hline & 20,70 & 2 \\
\hline \multirow[t]{5}{*}{$12 \mathrm{Y}$} & 20,70 & 5 \\
\hline & 20,95 & 1 \\
\hline & 20,85 & 10 \\
\hline & 20,80 & 1 \\
\hline & 20,90 & 3 \\
\hline \multicolumn{3}{|c|}{ Birds in the control condition } \\
\hline \multirow[t]{5}{*}{$29 \mathrm{~W}$} & 20,50 & 3 \\
\hline & 20,60 & 1 \\
\hline & 20,65 & 3 \\
\hline & 20,90 & 8 \\
\hline & 20,70 & 5 \\
\hline \multirow[t]{2}{*}{$66 \mathrm{Y}$} & 20,60 & 19 \\
\hline & 20,65 & 1 \\
\hline \multirow[t]{2}{*}{$62 \mathrm{R}$} & 20,60 & 3 \\
\hline & 20,65 & 17 \\
\hline \multirow[t]{3}{*}{$52 \mathrm{~W}$} & 20,60 & 3 \\
\hline & 20,80 & 10 \\
\hline & 20,65 & 7 \\
\hline
\end{tabular}

During reassignment training, pigeons were trained with only the $0^{\circ}$ view of each object; that view was associated with the peck requirement that was opposite to the peck requirement in original training. So, if the barrel was associated with FR20 during original training, then it was associated with FR60 during reassignment training. A randomized block design was used, in which each block consisted of ten presentations of the $0^{\circ}$ view of the barrel and the brick, for a total of 20 trials in a single block. There were eight blocks in each daily session for a total of 160 trials. A pigeon remained in reassignment training until it attained a peck rate to the stimulus associated with the low requirement that was 1.0 pecks per $\mathrm{s}$ higher than the peck rate to the stimulus associated with the high requirement and maintained this performance for 3 consecutive days.

During the testing phase, the pigeons continued to receive trials with the $0^{\circ}$ view of each geon; the peck requirements arranged in reassignment training remained in force. The testing stimuli comprised the four non-reassigned views $\left(72^{\circ}\right.$, $144^{\circ},-144^{\circ}$, and $-72^{\circ}$ ) of both objects; these views had been given to the birds in the Reassignment condition during the 
original training phase, but they were withheld during the reassignment training phase. In addition, the $0^{\circ}$ reassigned view was given as a testing stimulus, to provide equal numbers of testing trials for each view. Either the low or the high peck requirement was randomly chosen for each testing trial, so that the birds were unable to benefit from explicit training on these trials. Testing sessions were alternated with reassignment training sessions during the testing phase to maintain performance on the reassigned stimuli. During the intervening reassignment training sessions, a pigeon was required to maintain the 1.0 peck per s difference between the peck requirements; if it did not, then it was returned to reassignment training until it again met the criterion for at least one session.

Testing sessions began with ten warm-up trials (five presentations of the $0^{\circ}$ view for each of the two objects). The remainder of the testing session followed a randomized block design. Each block consisted of 32 trials with 30 of the trials involving the reassigned training views (15 presentations of the $0^{\circ}$ view of the two objects) and the remaining two trials involving the testing stimuli. The two testing stimuli were chosen randomly without replacement from the total pool of ten testing stimuli (the $72^{\circ}, 144^{\circ}, 0^{\circ},-144^{\circ}$, and $-72^{\circ}$ views of each object). There were five blocks in each daily session for a total of 170 trials (ten warm-up trials with the reassigned peck ratios, 150 training trials with the reassigned peck ratios, and ten testing trials with randomly chosen ratios). Each bird continued in testing until it had received 20 testing sessions.

\section{Results and discussion}

The pigeons in the Reassignment and Control conditions spent a mean of 25 days and 16 days in original training, respectively; this disparity suggests that learning to associate the different peck requirement to five views of each object in the Reassignment condition was more difficult than associating the requirement with a single view of each object. The pigeons in both the Reassignment and Control conditions spent a mean of 7 days in reassignment training. Testing sessions were alternated with reassignment sessions until a bird had completed a total of 20 testing sessions. Reassignment sessions were given during the testing phase to maintain performance to the reassigned stimuli. The minimum number of days in testing was 39 (20 testing sessions and a minimum of 19 reassignment sessions). However, if a pigeon did not meet the criterion during a reassignment session $($ criterion $=$ peck rate to the low FR stimulus at least 1.0 pecks per s higher than the peck rate to the high FR stimulus), then it would remain in reassignment training until it once again met the criterion. As a result of this requirement, during the testing phase, pigeons in the Reassignment condition had a mean of 46 reassignment sessions and pigeons in the Control condition had a mean of 34 reassignment sessions (the minimum number of sessions required if the criterion were always met would have been 19).
Again, these data suggest that performance was more difficult to maintain in the Reassignment condition.

Five birds did not complete the experiment. Two pigeons were replaced because they failed to meet criterion in original training after 60 sessions. Two additional pigeons were replaced because they did not meet criterion in reassignment training after 25 sessions; this criterion was set in reassignment training to alleviate memory problems due to long delays between original training and testing. Finally, a fifth pigeon was replaced because it completed only 1 day of testing followed by 23 days of reassignment training, again creating an extended delay between original training and the remainder of the testing sessions. In total, eight of the 13 pigeons that started the experiment completed all three phases; the results from those pigeons were analyzed.

Only data from the testing phase are presented here. To analyze these data, we used the difference score between the peck rates to the stimuli associated with a high number of pecks and to the stimuli associated with a low number of pecks. Using the difference score minimizes differences in overall response rate among pigeons.

To calculate the difference score, for each of the 20 testing sessions, we subtracted the mean peck rates to both types of stimuli at the $0^{\circ}$ view. For the remaining views, we combined the $-72^{\circ}$ and $72^{\circ}$ views and the $-144^{\circ}$ and $144^{\circ}$ views of each object prior to subtracting the high peck-requirement stimuli from the low peck-requirement stimuli; the direction of rotation was arbitrarily chosen and was not informative when comparing data between objects.

If the pigeons in the Reassignment condition maintained a high peck rate to the object associated with FR20 and a low peck rate to the object associated with FR60 - even to the withheld views - then the difference scores at $72^{\circ}$ and $144^{\circ}$ should not be significantly smaller than the difference score at $0^{\circ}$. Thus, the peck rate difference scores should not be significantly different from one another at the $0^{\circ}, 72^{\circ}$, and $144^{\circ}$ views. Figure 7 shows the difference scores for the Reassignment condition and the Control condition. It is evident from Fig. 7 that the pigeons did not respond similarly at the different distances from the reassigned view in the Reassignment condition. The difference between the peck rates for the objects associated with high and low peck requirements was much smaller for the withheld views than for the reassigned view. For the reassigned $\left(0^{\circ}\right)$ view the mean difference score was 1.2 , whereas for the withheld views the difference scores were much smaller $\left(72^{\circ}=0.6 ; 144^{\circ}=0.6\right)$. We can compare this pattern to that of the Control condition for which the results were quite similar: a difference score of 1.1 for the $0^{\circ}$ views, 0.8 for the $72^{\circ}$ views, and 0.4 for the $144^{\circ}$ views.

To statistically assess these observations, we first analyzed the data from the Reassignment condition alone. The peck rates to the testing stimuli were analyzed using a repeated 


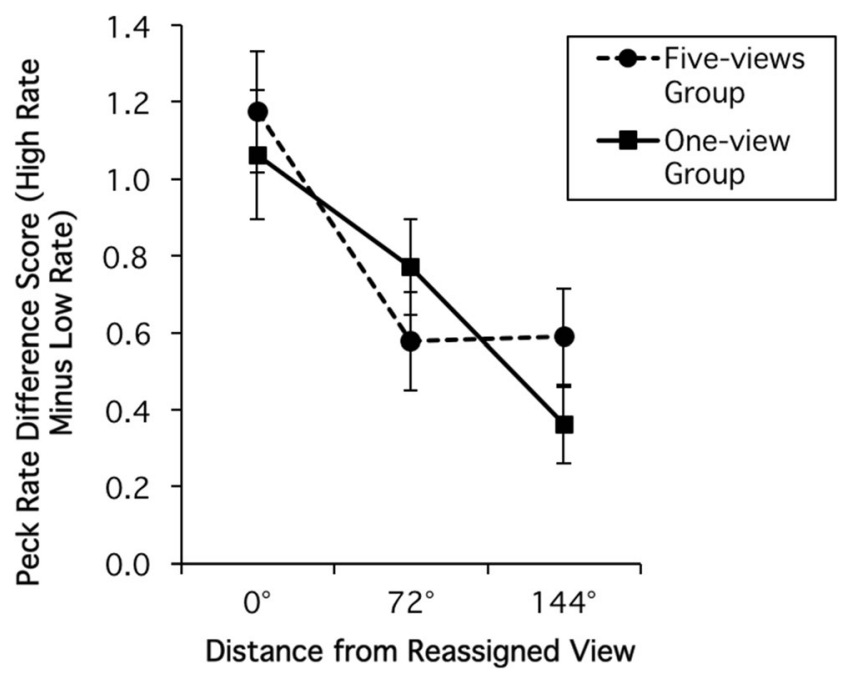

Fig. 7 The peck rate difference scores (response to high rate stimulus minus response to low rate stimulus) in Experiment 3 during the testing phase plotted as a function of distance from the reassigned view. Error bars represent $95 \%$ confidence intervals

measures, full factorial one-way ANOVA of Distance from Training View $\left(0^{\circ}, 72^{\circ}\right.$, and $\left.144^{\circ}\right)$. The peck rate difference scores were the dependent measure. The main effect of Distance from the Training View was significant $[F(2,6)=$ $\left.7.10, p<.05, \eta_{\text {partial }}^{2}=0.10\right]$. A planned comparison between the $0^{\circ}$ reassigned view and the view $72^{\circ}$ from the reassigned view (includes both the $72^{\circ}$ and $-72^{\circ}$ views) revealed a significant difference, $t=3.22, p<.05$. A planned comparison between the $0^{\circ}$ reassigned view and the view $144^{\circ}$ from the reassigned view was also significant, $t=3.16, p<.05$. These results indicate that there were robust disparities between the peck rates for the reassigned views and the withheld views of the objects.

Next, we compared the testing data for the Reassignment and Control conditions. Comparing the Reassignment condition to the Control condition was necessary to determine whether initial training with all five views in the Experimental condition had any effect on the pigeons' response rates to the four withheld views. The only procedural difference between the conditions was this initial training; the Control condition was trained with only one view and the Experimental condition was trained with 5 views. The data are presented in Fig. 7. Surprisingly, the pigeons in the Control condition exhibited a larger difference score than the Reassignment condition at the $72^{\circ}$ rotation. However, at the $144^{\circ}$ rotation, this pattern was reversed.

To explore these data in greater detail, the difference scores were analyzed using a repeated measures, full factorial analysis of variance (ANOVA) of View $\left(0^{\circ}, 72^{\circ}\right.$, and $\left.144^{\circ}\right)$ as within-participant variables and Condition (reassignment and control) as a between-participants variable. The main effect of View was significant $\left[F(2,6)=14.44, p<0.001, \eta_{\text {partial }}^{2}=\right.$ $0.10]$, indicating that the pigeons did not respond identically to all views of the objects. The main effect of Condition was not significant $[F(1,6)=0.07, p>0.05]$, however, indicating that across all views, there was no overall disparity between the groups' difference scores. The interaction between View and Condition was also non-significant $[F(2,12)=2.40, p>0.05]$, indicating that there was no significant difference in the patterns of responding to the different views.

We also wanted to analyze Days 1 and 2 of the Reassignment condition in the testing phase separately to determine if repeated testing over many days might wash out any effects that may have been evident early in testing. Figure 8 shows the first 2 days of testing plotted along with the full 20 days of testing. Again, we saw very little difference between peck rates disparities on the first 2 days of the experiment compared to the full 20 days of testing. The peck rates to the testing stimuli for Days 1 and 2 were analyzed using a repeated measures, full factorial one-way ANOVA of Distance from Training View $\left(0^{\circ}, 72^{\circ}\right.$, and $\left.144^{\circ}\right)$. The peck rate difference scores were the dependent measure. The main effect of Distance from the Training View was significant $\left[F(2,6)=6.45, p<.05, \eta_{\text {partial }}^{2}=0.15\right]$. Thus, there appear to be no appreciable differences between the results from Days 1 and 2 and those from the full 20 days.

In Experiment 3, we explored whether changing the response properties of the reassignment procedure would produce results that were consistent with previous recognition studies using these stimuli (Peissig et al., 2000a, 2000b). Astley and Wasserman (1999) demonstrated that paradigms which directly affect the delay or probability of reinforcement are extremely effective in supporting the formation of equivalence classes in pigeons. These types of manipulations, which directly affect reinforcement, may simply be more

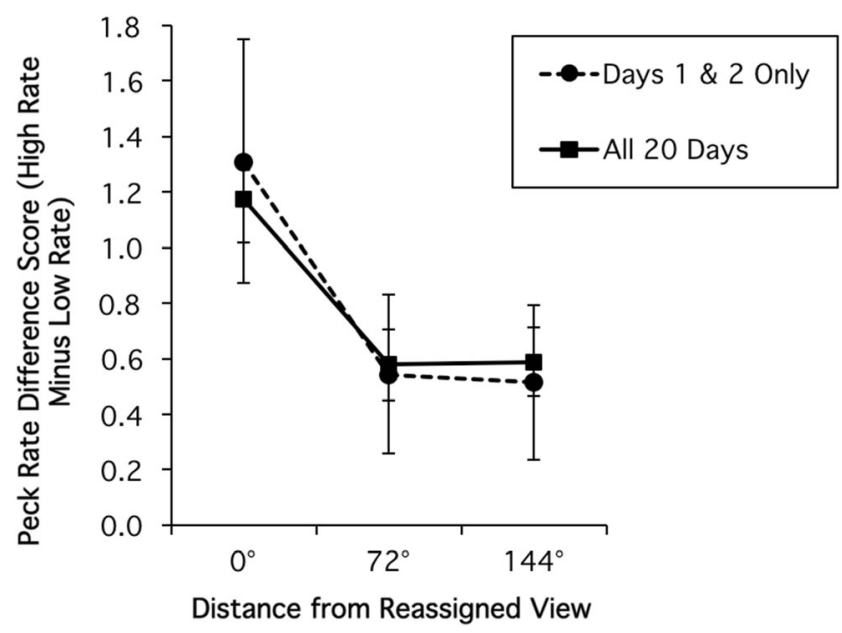

Fig. 8 The peck rate difference scores (response to high rate stimulus minus response to low rate stimulus) in the Reassignment condition for days 1 and 2 of the testing phase of Experiment 3 plotted along with the scores for days $1-20$ of the testing phase. The data are plotted as a function of distance from the reassigned view. Error bars represent $95 \%$ confidence intervals 
salient to the pigeon compared to responses such as choice button selections. To determine whether the results of Experiments 1 and 2 were due to the specific training parameters, we used an associative mediator that may be more salient to the pigeon than a particular button response - the number of pecks required to earn reinforcement.

It is evident from the results of this experiment that the data from Experiments 1 and 2 did not merely reflect the low salience of the choice button response for the pigeon. In Experiment 3, we used different work requirements in an attempt to strengthen the associations learned during the course of the experiment. Nevertheless, pigeons trained with five views (Reassignment condition) did not generalize the reassigned response to the withheld views to any greater degree than did pigeons trained with only one view (Control condition). Consistent with the results of Experiments 1 and 2, the results of this experiment suggest that pigeons do not form a general object concept that they are then able to use to mediate the changes in response contingencies in the reassignment procedure.

\section{Experiment 4}

In Experiments 1, 2, and 3, we were unable to find evidence that the pigeons generalized the new, reassigned response to the withheld views to an extent that is any greater than would be expected based on novel stimulus generalization. At this point, it may be tempting to conclude that these results are simply due to limitations on the pigeon's ability to form general object concepts. However, it is unclear whether humans would fare any better in a reassignment experiment without the benefit of explicit instructions.

There are studies which suggest that humans would readily generalize the newly trained response to non-reassigned views. Gelman and Markman (1986) tested whether young children would be biased to respond based on appearance or on category membership when these conflicted. For example, would children predict that a shark breathes more like a tropical fish (in the same category) or a dolphin (more visually similar)? They found that 4-year-olds were able to respond based on the category rather than visual appearance. These findings indicate that, even at a young age, humans readily form general concepts and accurately apply these concepts.

Although studies like Gelman and Markman (1986) suggest that it is likely that humans would generalize in a reassignment task, our specific methodology and stimuli have never been tested with humans. In the studies presented here, we were not testing the properties of category membership (e.g., does a shark breathe like a dolphin or a fish?). Rather, we were examining how different object views are treated when one view has been reassigned to a new label. Also, just like the pigeons, we wanted the humans in our study to have to complete the task without explicit instructions, and instead learn the correct responses via trial-and-error.

So, in Experiment 4, we gave people a task that was nearly identical to the one that we gave pigeons in Experiment 1, and involving the same stimuli (see Fig. 2). We used key presses on a standard keyboard instead of pecks to a touchscreen; the same four key presses were used during original training and reassignment training. People were not given any explicit instructions regarding the nature of the task; they were only instructed to press keys when a stimulus was presented and told they would have to guess to determine the correct responses.

\section{Method}

Participants A total of 20 undergraduate students from the Psychology Subject Pool at California State University, Fullerton received credit in their psychology courses in exchange for their participation.

Apparatus The computer-based tasks were carried out with the use of three 20-in iMac ${ }^{\circledR}$ (Apple Incorporated, Cupertino, CA, USA) computers. The computer tasks were executed using Superlab 4.0.7b (Cedrus Corporation, San Pedro, CA, USA).

Stimuli The stimuli were the same as those used in Experiment 1: an arch, a barrel, a brick, and a wedge that varied from one another by a variety of non-accidental properties of their generalized cone characterizations (Biederman, 1987). The geons were rotated in depth along a horizontal axis in $72^{\circ}$ intervals to yield 5 different views of each geon (see Fig. 2).

Procedure Prior to the beginning of the study, people were instructed to choose one of four keys on the keyboard in response to the stimulus: the "c" and " $\mathrm{v}$ " keys for the left hand and the " $\mathrm{n}$ " and " $\mathrm{m}$ " keys for right hand. These four keys were each labeled with a different colored sticker (with the letters still visible) to help participants find them easily. Participants were instructed to respond as quickly and accurately as possible and were also informed that they would have to guess initially to determine the correct response. At the beginning of a trial, the center display area was illuminated with a black cross centered on a white background for $500 \mathrm{~ms}$. The fixation cross was followed by a single-geon stimulus presented in the center of the screen. After a correct choice, the stimulus was removed from the display area and an intertrial interval of 1,500 ms was given. After an incorrect choice, an "incorrect" tone (Basso.aiff which is a standard sound effect on Macintosh computers) was given and the stimulus was turned off. The participant then proceeded through one or more correction trials (repeating the incorrect trial until a 
correct choice was made). There was no limit on the number of correction trials given and these trials were not scored for analysis.

Experiment 4 comprised three phases: original training, reassignment training, and testing. In original training, people were taught to press one of the four different keyboard keys ("c", " $v$ ", " $\mathrm{n}$ ", and " $\mathrm{m}$ ") in response to all five views $\left(0^{\circ}, 72^{\circ}\right.$, $144^{\circ},-144^{\circ}$, and $-72^{\circ}$, created by rotating the objects in depth in the horizontal axis) of each of the four single-geon stimuli (see Fig. 2). Keyboard assignments were counterbalanced across the three computers using a partial Latin-square design. We used a randomized block design for original training; each block consisted of one presentation of each of the five views of each of the four geons, yielding a total of 20 trials, each of which was presented twice. In reassignment training, one view (the $0^{\circ}$ view) of each of the four geons was reassigned to a new keyboard response. The four remaining views of each geon were withheld during reassignment training. Key reassignment was again counterbalanced across the three computers. For reassignment training, we used a randomized block design; each block consisted of ten presentations of the $0^{\circ}$ view of each of the four geons for a total of 40 trials in the reassignment phase. The same procedure for correct and incorrect trials used in the training phase was used in the reassignment phase.

In the testing phase, trials with the tested stimuli were nondifferentially reinforced; that is, the participant received no feedback, and all trials ended in a manner identical to correct trials in the preceding two phases. The instruction screen prior to starting the testing phase informed participants that they would receive no feedback. The testing phase followed a randomized block design and contained a total of 40 trials, including each of the four reassigned views and the remaining 16 withheld views, each presented twice.

\section{Results and discussion}

Two participants were excluded from analysis; these participants did not achieve $80 \%$ or greater accuracy in the second half of original training. All of the 18 remaining participants achieved $80 \%$ or above accuracy in the second half of original training. The data were collapsed across the $-72^{\circ}$ and $72^{\circ}$ rotations and the $-144^{\circ}$ and $144^{\circ}$ rotations across all four geons. The mean choice accuracy for each of the three views was $92 \%$. It is evident from these data that people showed highly similar performance to the reassigned and withheld views.

In order to confirm these initial observations, we analyzed choice accuracy using a repeated measures, full factorial ANOVA of Geon (arch, barrel, brick, and wedge) by View $\left(-144^{\circ},-72^{\circ}, 0^{\circ}, 72^{\circ}\right.$, and $\left.144^{\circ}\right)$. The main effect of Geon was not significant $[F(3,51)=1.28, p>0.05]$, indicating that the people's choice accuracy for the different geons did not differ significantly from one another. The main effect of View was non-significant $[F(4,68)=0.84, p>0.05]$, indicating that people responded similarly to the five different views. The interaction of Geon by View was not significant, $[F(12,204)$ $=1.17, p>0.05]$; this result indicated that people responded similarly to the different views for all four geons.

The results with human participants indicate that, as previous studies suggested, people more readily form equivalence classes consisting of different views of the same object than do pigeons. Thus, it appears that the fundamental issue that is raised by our first three experiments is not a problem with the reassignment paradigm itself, but rather concerns what pigeons learn under this training paradigm and/or the way in which pigeons store multiple views of an object. In this connection, it is important to consider the possibility that people may have used a verbal label for each geon, which they could then easily use to help them associate the new, reassigned response to all views of the geons. In order to eliminate this possibility, however, we would need to create highly variable categories, so that a single verbal label would not be possible, something that could be explored in future comparative studies.

\section{General discussion}

We conducted four experiments that used Lea's (1984) reassignment procedure in both pigeons and humans to better understand how object concepts are utilized. In Experiment 1, we trained pigeons with five views of four different simple objects (geons). We then reassigned a single view of each geon to a new response. Finally, we showed the withheld views to the pigeons to determine the extent to which they generalized the new response to the untrained views. If the birds exhibited response accuracies that were similar for both reassigned and non-reassigned views, then such generalization would have indicated that pigeons form a flexible general concept for each object that is similar to the object representations that are formed by normal adult humans. If that were the case, then reassigning the response to one view of that object should have automatically led to a change in responding to all of the remaining views. This was not the result we found. Rather, the new response did not generalize to the remaining views any more than would have been expected had the birds never been trained with those additional views.

In Experiments 2 and 3, the reassignment procedure was further explored in pigeons by attempting to make the connection between the new response to the withheld views of the same object more salient. Again, the pigeons did not show similar responding to both the reassigned and the withheld views. Finally, in Experiment 4, we tested humans with the same stimuli and task as in Experiment 1 to determine whether humans would more readily generalize the new response than 
did pigeons. As expected, people did robustly generalize the new response to the withheld views.

It is possible that the pigeons performed a very simple visual analysis, such as pixel similarity, rather than object or image similarity (which should be greater among different views of the same object than to different views of different objects) to determine how object views should be processed. Thus, if there were more pixel overlap between a particular view of the arch and a particular view of the brick, then the pigeons' key responses may have been biased. In previous studies, we have analyzed this possibility and found that pixel similarity cannot account for the typical patterns of generalization such as those reported here (Peissig et al. 2000a).

Taken together, these data suggest that people form general object concepts and respond similarly to all views of the same object. For pigeons, however, another process or mechanism may be interfering with such generalization. It may be that that pigeons form individual representations for each of the trained views, and, depending on the training conditions, the connection among these different views of the same object might be made to be stronger or weaker. Previous studies have shown that pigeons generalize more readily to views of the same object than they do to views of different objects (Peissig et al. 2000b). This finding held even though the pigeons had been trained with only one view of the target object as the $\mathrm{S}+$, while different views of the target object as well as diverse views of a different object were each trained as S-s.

Our three different versions of the reassignment paradigm failed to show that pigeons form a robust, flexible general object representation for each object. One possible explanation for our present findings is that the reassignment procedure is inherently flawed. However, in Experiment 4, we were able to use the procedure successfully with humans, so we can rule out this possibility.

Another explanation is that the reassignment procedure does not work when given to pigeons. Certainly, the literature does contain some examples of failures of the procedure to reveal concept formation (Bhatt \& Wasserman, 1989; Von Fersen \& Lea, 1990), but there are also several examples of successes (Delius, Ameling, Staddon, \& Lea, 1995; Vaughan, 1988; Wasserman, DeVolder, \& Coppage, 1992). It may be important to note that, when the reassignment procedure has been successful, it has often (but not always) involved repeated contingency reversals (Delius et al. 1995; Vaughan, 1988). These reversals could act as a kind of tutelage for the pigeons, effectively instructing them that they are to associate the stimuli with one another and respond in kind. This suggests that pigeons have difficulty with changing contingencies and require additional training to be able to quickly accommodate these changes.

One limiting factor may be the number of stimuli that are given during reassignment training. Perhaps because only one exemplar from each of the four categories was shown during reassignment training, inadequate information was provided to the pigeon as to the adequacy of the stimulus representing all four of the remaining members of the category. This limitation may be an indication of weaker binding of different views to a general object concept for pigeons. After all, none of the category members was new; each had previously been associated with the same response to the same criterion level, and therefore should have been substitutable for one another. Indeed, independent evidence suggests that members of each of the object classes look more like one another than they resemble members of the other object classes (Peissig et al., 2002; Peissig et al., 2000b).

Another possible explanation for the pigeon data is that the birds are behaving as do humans with prefrontal cortex damage or very young children when faced with a sudden reversal or change in contingencies (Kendler \& Kendler, 1962). For example, researchers have shown that people with some types of prefrontal cortex damage do poorly on problems such as the Wisconsin Card Sorting Task (Bornstein, 1986; Milner, 1963; Stuss et al., 2000). These individuals may initially understand the card sorting task, however they later do poorly because they do not know how to respond when the contingencies are changed. Similarly, Kendler and Kendler (1962) discuss research which shows that children at the age of four have difficulty with reversals of behavioral contingencies; however, by the age of seven children are able to rapidly adjust to reversals. Their results suggest a developmental shift in humans exposed to these types of tasks. If these decisional processes are related to why the pigeons sometimes perform poorly in the reassignment procedure, then we might expect that young children (age 4 years and under) would behave more like pigeons than adult humans in our version of the reassignment task.

In conclusion, our study may suggest an important difference in the structure of object representations between humans and pigeons (for more on this possibility, see Soto \& Wasserman, 2014; Wasserman \& Biederman, 2012). Pigeons may form individual representations for each of the trained views, and, depending on the training conditions, the connection among these different views of the same object might be stronger or weaker. Alternatively, pigeons' decision-making abilities may be unable to adjust to abrupt changes in response contingency; they may only be able to respond appropriately after many reversals or extensive response-switch training.

Based on the results of several other studies (Peissig et al. 2000a \& 2000b; Peissig et al. 2002; Wasserman \& Biederman, 2012), it is obvious that whatever form the object representation takes, it is sufficiently flexible to allow for fairly broad generalization under a large number of learning and testing conditions. However, deploying the reassignment procedure with multiple object views may push pigeons to the limit in displaying that flexibility. 
Acknowledgments This research was supported by a National Institute of Mental Health Grant, MH 47313. A portion of this report was included in the doctoral dissertation of Jessie J. Peissig at The University of Iowa. We would like to thank C. Antes, T. Barclay, M. Prier, B. J. Terrones, O. Lazareva, L. Castro, A. Frank, and M. Miner for their assistance in collecting and analyzing these data.

\section{References}

Astley, S. L., Peissig, J. J., \& Wasserman, E. A. (2002). Superordinate categorization via learned stimulus equivalence: Quantity of reinforcement, hedonic value, and the nature of the mediator. Journal of Experimental Psychology: Animal Behavior Processes, 27, 252-268.

Astley, S. L., \& Wasserman, E. A. (1992). Categorical discrimination and generalization in pigeons: All negative stimuli are not created equal. Journal of Experimental Psychology: Animal Behavior Processes, 27, 193-207.

Astley, S. L., \& Wasserman, E. A. (1998). Novelty and functional equivalence in superordinate categories in pigeons. Animal Learning and Behavior, 26, 125-138.

Astley, S. L., \& Wasserman, E. A. (1999). Superordinate category formation in pigeons: Association with a common delay or probability of food reinforcement makes perceptually dissimilar stimuli functionally equivalent. Journal of Experimental Psychology: Animal Behavior Processes, 25, 415-432.

Bhatt, R. S., \& Wasserman, E. A. (1987). Choice behavior of pigeons on progressive and multiple schedules: A test of optimal foraging theory. Journal of Experimental Psychology: Animal Behavior Processes, 13, 40-51.

Bhatt, R. S., \& Wasserman, E. A. (1989). Secondary generalization and categorization in pigeons. Journal of the Experimental Analysis of Behavior, 52, 213-224.

Bhatt, R. S., Wasserman, E. A., Reynolds, W. F., Jr., \& Knauss, K. S. (1988). Conceptual behavior in pigeons: Categorization of both familiar and novel examples from four classes of natural and artificial stimuli. Journal of Experimental Psychology: Animal Behavior Processes, 14, 219-234.

Biederman, I. (1987). Recognition-by-Components: A theory of human image understanding. Psychological Review, 94, 115-147.

Biederman, I. (2001). Recognizing depth-rotated objects: A review of recent research and theory. Spatial Vision, 13, 241-253.

Bornstein, R. A. (1986). Contribution of various neuropsychological measure to detection of frontal lobe impairment. International Journal of Clinical Neuropsychology, 8, 18-22.

Bülthoff, H. H., \& Edelman, S. (1992). Psychophysical support for a twodimensional view interpolation theory of object recognition. Proceedings of the National Academy of Science USA, 89, 60-64.

Cimpian, A., \& Erickson, L. C. (2012). Remembering kinds: New evidence that categories are privileged in children's thinking. Cognitive Psychology, 64, 161-185.

Delius, J. D., Ameling, M., Lea, S. E. G., \& Staddon, J. E. R. (1995). Reinforcement concordance induces and maintains stimulus associations in pigeons. Psychological Record, 45, 283-297.

DiPietro, N. T., Wasserman, E. A., \& Young, M. E. (2002). Effects of occlusion on pigeons' visual object recognition. Perception, 31, 1299-1312.

Felton, M., \& Lyon, D. O. (1966). The post-reinforcement pause. Journal of the Experimental Analysis of Behavior, 9, 131-134.

Gelman, S. A., \& Markman, E. M. (1986). Categories and induction in young children. Cognition, 23, 183-209.

Gibson, B. M., Wasserman, E. A., Frei, L., \& Miller, K. (2004). Recent advances in operant conditioning technology: A versatile and affordable computerized touchscreen system. Behavior Research Methods, Instruments, \& Computers, 36, 355-362.
Herrnstein, R. J., \& Loveland, D. H. (1964). Complex visual concept in the pigeon. Science, 146, 549-551.

Kendler, H. H., \& Kendler, T. S. (1962). Vertical and horizontal processes in problem solving. Psychological Review, 69(1), 1-16.

Lea, S. E. G. (1984). In what sense do pigeons learn concepts? In H. L. Roitblat, T. G. Bever, \& H. S. Terrace (Eds.), Animal cognition (pp. 263-276). Hillsdale: Erlbaum.

Mallot, R. N., \& Siddal, J. N. (1972). Acquisition of the people concept in pigeons. Psychological Reports, 31, 3-13.

Martin-Malivel, J., Mangini, M. C., Fagot, J., \& Biederman, I. (2006). Do humans and baboons use the same information when categorizing human and baboons faces. Psychological Science, 17, 599-607.

Milner, B. (1963). Effects of different brain lesions on card sorting: The role of the frontal lobes. Archives of Neurology, 9, 100-110.

Murphy, G. L. (2010). What are categories and concepts? In D. Mareschel, P. C. Quinn, \& S. E. G. Lea (Eds.), The making of human concepts (pp. 11-28). New York: Oxford University Press.

Peissig, J. J., \& Tarr, M. J. (2007). Object recognition: Do we know more today than we did twenty years ago? In S. T. Fiske, A. E. Kazdin, \& D. L. Schacter (Eds.), Annual review of psychology (Vol. 58, pp. 7596). Palo Alto: Annual Reviews Press.

Peissig, J. J., Young, M. E., Wasserman, E. A., \& Biederman, I. (2000a). Seeing things from a different angle: The pigeon's discrimination of single geons rotated in depth. Journal of Experimental Psychology: Animal Behavior Processes, 26, 115-132.

Peissig, J. J., Young, M. E., Wasserman, E. A., \& Biederman, I. (2000b). The pigeon's perception of depth-rotated shapes. In J. Fagot (Ed.), Picture perception in animals (pp. 37-70). East Sussex: Psychology Press, Ltd.

Peissig, J. J., Wasserman, E. A., Young, M. E., \& Biederman, I. (2002). Learning an object from multiple views enhances its recognition in an orthogonal rotational axis in pigeons. Vision Research, 42, 2051-2062.

Siegel, R. K., \& Honig, W. K. (1970). Pigeon concept formation: Successive and simultaneous acquisition. Journal of the Experimental Analysis of Behavior, 13, 385-390.

Sigala, N., \& Logothetis, N. K. (2002). Visual categorization shapes feature selectivity in the primate temporal cortex. Nature, 415(6869), 318-320.

Soto, F. A., \& Wasserman, E. A. (2012). Visual object categorization in birds and primates: Integrating behavioral, neurobiological, and computational evidence within a "general process" framework. Cognitive, Affective, \& Behavioral Neuroscience, 12, 220-240.

Soto, F. A., \& Wasserman, E. A. (2014). Mechanisms of object recognition: What we have learned from pigeons. Frontiers in Neural Circuits. doi:10.3389/fncir.2014.00122

Spetch, M. L., \& Friedman, A. (2003). Recognizing rotated views of objects: Interpolation versus generalization by humans and pigeons. Psychonomic Bulletin and Review, 10, 135-140.

Spetch, M. L., \& Friedman, A. (2006). Pigeons see correspondence between objects and their pictures. Psychological Science, 17, 966-972.

Stuss, D. T., Levine, B., Alexander, M. P., Hong, J., Palumbo, C., Hamer, L. ... Izukawa, D. (2000). Wisconsin Card Sorting Test performance in patients with focal frontal and posterior brain damage: Effects of lesion location and test structure on separable cognitive processes. Neuropsychologia, 38, 388-402.

Vaughan, W. (1988). Formation of equivalence sets in pigeons. Journal of Experimental Psychology: Animal Behavior Processes, 14, 36-42.

Von Fersen, L., \& Lea, S. E. G. (1990). Category discrimination by pigeons using 5 polymorphous features. Journal of the Experimental Analysis of Behavior, 54, 69-84.

Wasserman, E. A., \& Bhatt, R. S. (1992). Conceptualization of natural and artificial stimuli by pigeons. In W. K. Honig \& J. G. Fetterman (Eds.), Cognitive aspects of stimulus control (pp. 203-223). Hillsdale: Erlbaum. 
Wasserman, E. A., \& Biederman, I. (2012). Recognition by components: A bird's eye view. In O. F. Lazareva, T. Shimizu, \& E. A. Wasserman (Eds.), How animals see the world. New York: Oxford University Press.

Wasserman, E. A., DeVolder, C. L., \& Coppage, D. J. (1992). Nonsimilarity-based conceptualization in pigeons via secondary or mediated generalization. Psychological Science, 3, 374-379.
Zentall, T. R. (2012). Working memory in animals. In T. R. Zentall \& E. A. Wasserman (Eds.), Oxford handbook of comparative cognition (pp. 215-238). New York: Oxford University Press.

Zentall, T. R., Wasserman, E. A., \& Urcuioli, P. J. (2014). Associative concept learning in animals. Journal of the Experimental Analysis of Behavior, 101, 130-151. 